\title{
Temporally convoluted Gaussian equations for chromatographic peaks
}

\author{
Su-Cheng Pai* \\ Division of Marine Chemistry, Institute of Oceanography, National Taiwan University, P.O. Box 23-13, Taipei, Taiwan
}

Received 9 July 2003; received in revised form 7 November 2003; accepted 19 November 2003

\begin{abstract}
Both spatial and temporal peaks that are produced by the discrete parcel model can be mathematically approximated by Gaussian functions, but the transformation from a spatial pattern to a temporal image requires a convolution treatment. A first-order convolution is given for temporal peaks under a linear isotherm, whereas a second-order convolution is proposed for those under non-linear isotherms. Numerical tests show that the peak shapes generated by the proposed temporally convoluted Gaussian equations (TCG) match perfectly with those obtained by the discrete parcel model. Although the full TCG equation may be quite complicated, it can be made easier by a recursion calculation technique, and a group of peak curves can be plotted simultaneously on computer worksheet. The results also suggest that the temporal distortion effect should be predominately considered, in addition to those known-to-exist spatial effects, for explaining the peak asymmetry. (c) 2004 Elsevier B.V. All rights reserved.
\end{abstract}

Keywords: Temporal convolution; Gaussian equations; Peak shape; Parcel model

\section{Introduction}

\subsection{Peak asymmetry}

Peak asymmetry has been puzzling chromatographers for many years. To solve the skewed peak shape problem, two opposing approaches have been consistently proceeded [1]: (i) the derivation from physical models; (ii) empirical peak shape simulation by mathematical functions. In the former approach, the peak shape is produced by setting up physical factors and solving the differential equations for a tubular flow system. It is generally qualitative, providing probable reasons why and how a peak is skewed during the chromatographic process. A variety of physical mechanisms have been identified, and the most widely adopted reasons include: the non-linear isotherm that occurs in the column [2,3]; the solution derived from Fick's Law, in which the diffusivity is a three-dimensional function of time due to the multi-layered flow speed (parabolic flow pattern) in the tubular column [4,5]; and the extra column effects (defects from the injector, detector, connector, ..., etc.) that cause the unevenly distributed mass pattern [5-7]. However, these explanations are of little consequence to practical users, as

\footnotetext{
* Tel.: +886-2-23627358; fax: +886-2-23632912.

E-mail address: scpai@ntu.edu.tw (S.-C. Pai).
}

the instrumental conditions vary from one laboratory to another. The users would require a more quantitative way for simulating the experimental chromatogram so as to obtain better separation results.

Numerous empirical functions have been suggested for the peak curve fitting. They have been evaluated and summarized by Di Marco and Bombi [8] into different categories. Among those, the Gaussian series with mathematical modifications (e.g. exponential or polynomial) are probably the ultimate choice for constructing chromatographic peaks [9-14]. There is no doubt that the Gaussian function can provide a solid basis for the curve-fitting purpose, but the mathematical treatments (e.g. polynomial terms) on the peak standard deviation terms are diverse and empirical. As a consequence, even though the modified Gaussian functions can indeed fit most experimental peak curves, their physical meanings are vague or not quite persuasive. The connection between the two approaches (theoretical derivation and empirical fitting) has not been well established.

\subsection{The missing link}

One important factor, the temporal distortion effect, has long been ignored by both communities. This effect, which is generated due to the non-simultaneous detection at a fixed position, can produce significant false tailings in flow injection analysis [15]. It can also cause a similar peak-image 
twisting in chromatography, which has been demonstrated by a lately published discrete parcel model [16]. In that model, the temporal peak data produced are plotted along the time coordinate, to distinguish from the spatial peak shapes which are plotted on the longitudinal axis. Various temporal peaks (symmetrical, tailing or fronting) are generated; even though they are spatially symmetrical throughout. Thus, the difference (or residue) between a pair of normalized spatial and temporal peaks gives the net "temporal distortion". It is very probable that much of the skewed fraction of the observed peaks is induced by this effect, although a direct experimental verification is not yet available.

It is the author's opinion that all physical models should be further modified to include the temporal distortion effect. On the other hand, when using mathematical functions, the curve-fitting would be much better if standing on a more reasonable platform to endorse the same effect. The link between the two approaches might well be the "spatial-temporal convolution".

\subsection{The convolution concept}

The temporal convolution effect is not a real mechanism that occurs inside a chromatographic column or in the injector and detector, but an axial transformation process if the broadened sample zone is observed by a single fixed detector. The relativity is the major concern, which has already been demonstrated in the previous parcel model, and it can be further explained by a systematic diagram as shown in Fig. 1. In this diagram a sample plug is injected into the column system, and the sample zone starts to expand promptly after leaving the injector. The zone broadening may involve many mechanisms, in-column or extra column, symmetrical or asymmetrical. Among these, "retention" is usually the main reason, in addition to a substantial fraction of dispersion-diffusion (high in GC but relatively low in HPLC), both are symmetrical in nature. There are several asymmetrical factors, including the non-ideal retention, the parabolic flow profile, as well as the extra column effect. The combination of all these effects results in the "spatial" concentration profile (noted as $C(n)$ or $m(n)$ ), which should be plotted along the longitudinal $(n)$ coordinate at a specific time.

The temporal image, however, cannot be obtained using a direct transformation from the longitudinal coordinate to a temporal scale by dividing the flow speed. The chromatographic peaks, laid on a temporal coordinate ( $\tau$, a dimensionless time in this study), are usually obtained by a detector located at the end of a column. The signal is recorded on a sequential basis when the sample zone passes through. Therefore, a temporal peak is a "composed image" of many minute segments of the spatial patterns at various times. Thus, the peak, denoted as $C(\tau)$ or $m(\tau)$, is a distorted one from its real spatial shape, and the difference in between is regarded as the "temporal distortion". The distortion is due to (i) the changing of the zone width and (ii) the changing

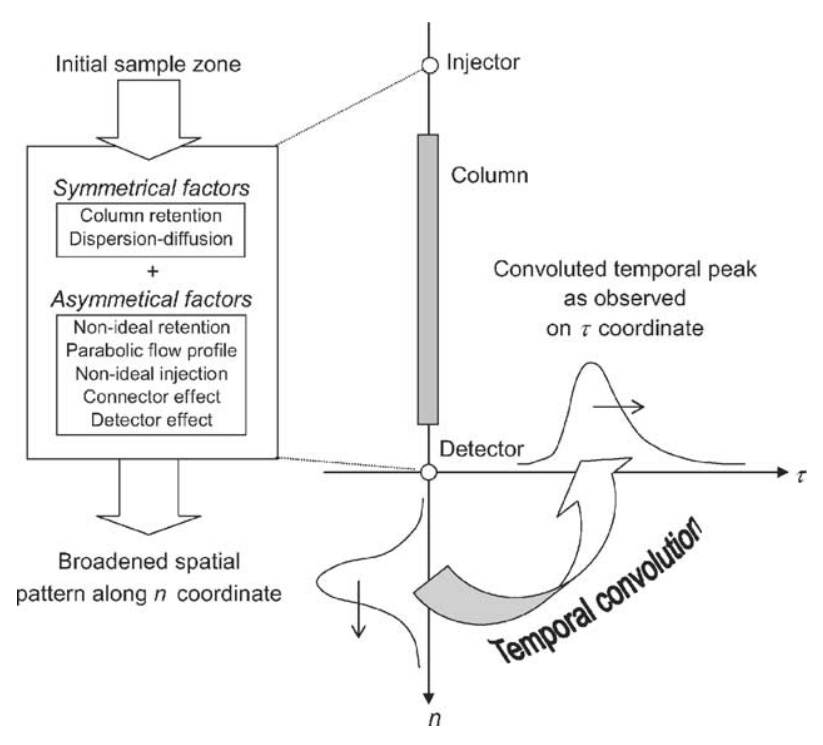

Fig. 1. Systematic diagram showing the possible broadening mechanisms during the chromatographic process and the concept of convolution. On the left, the initial sample zone is broadened by a number of mechanisms, mainly by "in-column" retention and dispersion-diffusion, which are symmetrical in nature. Several non-ideal and extra column effects may also contribute a small portion of the zone broadening, which might be asymmetrical. The resultant spatial pattern along the $n$ coordinate, if observed by a fixed detector, will be distorted on the temporal $\tau$ coordinate. The process is termed the "spatial-temporal axial-transformation convolution" or simply "temporal convolution".

of the migration speed during the passage through the detector. Its scale relates to the position of the observer and the isotherm programming rather than the physical mechanisms occurring in the column.

\subsection{Focus of this work}

The complete peak formation process (spatial band broadening plus temporal convolution) can be very complicated. For simplicity, these spatially induced asymmetrical factors are not considered in this study, and only the in-column retention behavior and its corresponding convolution process are emphasized. The broadening mechanism follows the posture of the previous discrete parcel model, and a temporally convoluted Gaussian function (TCG) is proposed to generate basic chromatographic peak shapes. The results will give clues to explain, "How a Gaussian-distributed pattern can be recorded to have various asymmetrical shapes". In other words, the TCG equations described here may be regarded as the continuous solution of the discrete parcel model.

\section{Theoretical}

\subsection{Basic Gaussian curve}

Since the entire convolution treatment is established on the Gaussian function, it is necessary to emphasize the 
meaning of each mathematical term. A basic Gaussian function, denoted as $y(x)$, consists of three components, i.e. the peak area $A$, the zone width or standard deviation $\sigma$, and the location $a$ on the $x$ coordinate. The mathematical expression is:

$y(x)=\frac{A}{\sqrt{2 \pi} \sigma} \mathrm{e}^{-[(x-a) /(\sqrt{2} \sigma)]^{2}}$

where $A, \sigma$ and $a$ are given numbers, independent of each other. The produced curve is a symmetrical pattern (Fig. 2).

For generating chromatographic peaks, these components may be related to each other in different ways (limiting cases). Three limiting derivatives will be described in this study, and they are also illustrated in Fig. 2.

Type I-The standard deviation $(\sigma)$ is correlated to the position $(a)$. This limiting case applies to most longitudinal zone broadenings. Since $A, a$ and $\sigma(a)$ are all fixed (or given values), the curve is still symmetrical. This type of equation is used to simulate the spatial distribution pattern along a column ( $x$-axis is the column length).
Type II-If $\sigma$ becomes a function of $x$, while $A$ and $a$ remain constant, the peak pattern may be distorted to appear asymmetrical. The mathematical treatment for this distortion is called the "convolution" along the $x$ coordinate. The peak summit may have a small shift from its expected position $a$. The Type II equation is used to simulate temporal chromatographic peaks under a linear condition. The $x$-axis here represents time (not length). The peak position remains constant since only the standard deviation term $\sigma(x)$ is variable of time.

Type III-If both $\sigma$ and $a$ are functions of $x$, then a double distortion occurs. This type of curve, while still maintaining the identical peak area $A$, will have various appearances depending on the conditions given. It could be nearly symmetrical, or having a prolonged or fronting tail, or have erratic shapes if the conditions are designated to be step-wise along the $x$ coordinate. This limiting type is applied to construct a chromatographic peak under a non-linear isotherm. Both the standard deviation $\sigma(x)$ and peak position $a(x)$ are variable along $x$ (time) axis, so the Gaussian peak curve

Basic Gaussian curve:

( $A, a, \sigma$ are independent)

$$
y(x)=\frac{A}{\sqrt{2 \pi} \sigma} e^{-[(x-a) /(\sqrt{2} \sigma)]^{2}}
$$

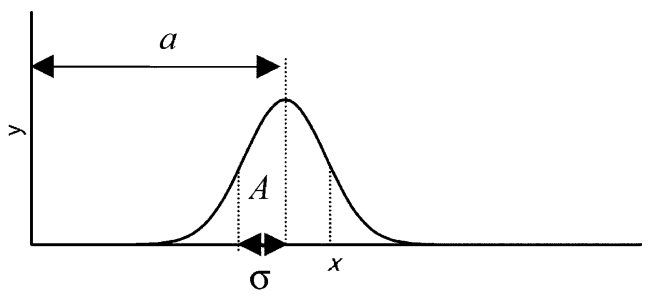

$$
\begin{aligned}
& \text { Limiting Gaussian Type-I: } \\
& (\sigma \text { is a function of } a) \\
& y(x)=\frac{A}{\sqrt{2 \pi} \sigma(a)} e^{-[(x-a) /(\sqrt{2} \sigma(a))]^{2}}
\end{aligned}
$$

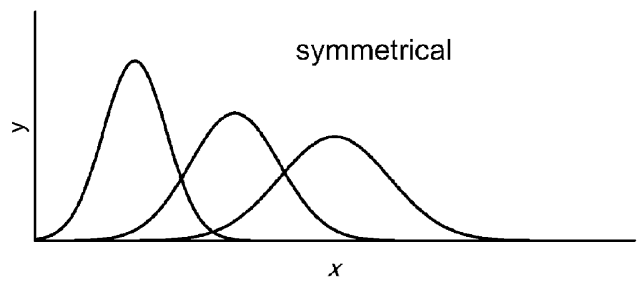

$$
\begin{aligned}
& \text { Limiting Gaussian Type-II: } \\
& (\sigma \text { is a function of } x) \\
& y(x)=\frac{A}{\sqrt{2 \pi} \sigma(x)} e^{-[(x-a)) /(\sqrt{2} \sigma(x))]^{2}}
\end{aligned}
$$

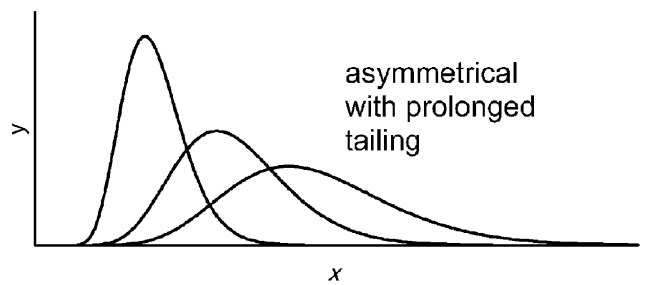

$$
\begin{gathered}
\text { Limiting Gaussian Type-III: } \\
\text { (Both } \sigma \text { and } a \text { are functions of } x \text { ) } \\
y(x)=\frac{A}{\sqrt{2 \pi} \sigma(x)} e^{-[(x-a(x)) /(\sqrt{2} \sigma(x))]^{2}}
\end{gathered}
$$

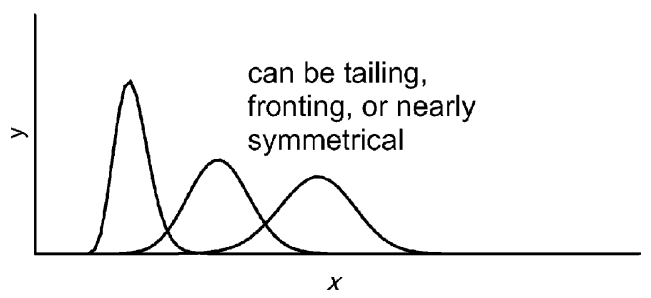

Fig. 2. Basic Gaussian equation $(y(x))$ and three limiting derivatives (Types I, II and III), which are used to construct chromatographic peaks. Examples are illustrated on the right. Symbols $A, a$ and $\sigma$ represent peak area, peak location and standard deviation, respectively. 
receives a "second-order" convolution on the observing coordinate.

The details of those peak parameters are described separately in the following sections.

\subsection{Peak parameters}

Most parameters and symbols used in this study are the same as those listed in the previous paper describing the discrete parcel matrix [16]. In that model, a column (length $L$ ) is divided into $n$ small sections, each one is $\Delta L$, so $L=$ $n \Delta L$; and the time duration $t$ is divided into many small time steps, each step is $\Delta t$ and $t=\tau \Delta t$. Thus the column operation is displayed on a dimensionless $n \times \tau$ integer matrix. Plotting data along the $n$ coordinate at selected $\tau$ gives the "spatial" mass distribution pattern, whereas plotting data along the $\tau$ coordinate at a fixed column length $n$ generates the "temporal" chromatographic peak.

The present study is a continuous one; therefore $n$ and $\tau$ may not necessarily be integers. These two symbols are re-defined as the "dimensionless length and time". To match the scale of the parcel model for comparison, the flow speed of the influent carrier is still defined to be $v=\mathrm{d} n / \mathrm{d} \tau=1$ in this paper.

\subsubsection{Partition equilibrium}

At a constant flow speed, the partition equilibrium of mass between the stationary and mobile phases at any specified $n$ and $\tau$ obeys the dynamic isotherm:

$k^{\prime \prime}(\tau)=\frac{m_{\mathrm{s}(n, \tau)}}{m_{\mathrm{m}(n, \tau)}}$

where $k^{\prime \prime}(\tau)$ is the dynamic partition ratio at a specific time $\tau$. If $k^{\prime \prime}(\tau)$ is constant throughout the elution period, it is called a linear isotherm. If not, it is a non-linear isotherm.

Each analyte has an initial $k_{0}^{\prime \prime}$ value. The $k^{\prime \prime}(\tau)$ value can be programmed to change during the elution. In gas chromatography, it is very common to set up a temperature program to serve this purpose. Thus, under a non-linear isotherm, $k^{\prime \prime}(\tau)$ can be expressed as an exponential function of time, with a decay constant $\lambda$ which is proportional to the rate of temperature elevation $(\mathrm{d} T / \mathrm{d} \tau)$.

$k^{\prime \prime}(\tau)=k_{0}^{\prime \prime} \mathrm{e}^{-\lambda \tau} \quad(\lambda \geqq 0)$

Examples are given in Fig. 3a and b.

\subsubsection{Flow speed and migration speed}

If the flow speed of the mobile carrier is $v=1$, the average migration speed of an analyte in the column at a given time $\tau$ is defined as $v_{\mathrm{m}}(\tau)$.

$v_{\mathrm{m}}(\tau)=\frac{1}{k^{\prime \prime}(\tau)+1}$

Examples are given in Fig. 3c.

\subsubsection{Peak position}

The peak position along the $n$ coordinate at a given time $\tau$ is denoted as $n_{\mathrm{p}}(\tau)$. It is obtained by integrating the migration speed $\left(\mathrm{d} n_{\mathrm{p}} / \mathrm{d} \tau=v_{\mathrm{m}}(\tau)\right)$ along time. Thus, under a linear isotherm $(\lambda=0)$, it can be expressed as:

$n_{\mathrm{p}}(\tau)=n_{\mathrm{s}}+\frac{\tau}{k_{0}^{\prime \prime}+1}$

where $n_{\mathrm{s}}$ is the longitudinal shift, an artifact in the parcel model due to the discrete calculation (to be 0.5 when the initial zone width $i=1$ ). It may be ignored when $\tau$ is large. Under a non-linear isotherm, the $k^{\prime \prime}(\tau)$ is no longer a fixed value (Eq. (3)), and the position becomes:

$n_{\mathrm{p}}(\tau)=n_{\mathrm{s}}+\tau+\frac{1}{\lambda} \ln \left(k_{0}^{\prime \prime} \mathrm{e}^{-\lambda \tau}+1\right)-\frac{1}{\lambda} \ln \left(k_{0}^{\prime \prime}+1\right)$

where $\lambda$ is the decay constant $(\lambda>0)$. The $n_{\mathrm{s}}$ term remains here although it might be negligible when $\tau$ is large.

\subsubsection{Spatial peak standard deviation}

The standard deviation on the $n$ coordinate is denoted as $\sigma_{n}(\tau)$. It is calculated by taking the square root of the initial zone variance plus the integration of the variance broadening rate (i.e. $\left.\mathrm{d} \sigma_{\mathrm{n}}(\tau)^{2} / \mathrm{d} \tau=k^{\prime \prime}(\tau) /\left(k^{\prime \prime}(\tau)+1\right)^{2}\right)$ at a specific time $\tau$ [17].

Under a linear isotherm, the standard deviation of a peak at a specific time $\tau$ is:

$\sigma_{\mathrm{n}}(\tau)=\sqrt{\sigma_{0}^{2}+\frac{k_{0}^{\prime \prime}}{\left(k_{0}^{\prime \prime}+1\right)^{2}} \tau}$

where $\sigma_{0}$ is the initial standard deviation of the injected sample zone, to be 0.34 in the parcel model (accounted by taking $34 \%$ of the possibility range of the initial zone width $i=1$ ).

Under a non-linear isotherm, when $k^{\prime \prime}(t)=k_{0}^{\prime \prime} \mathrm{e}^{-\lambda \tau}$, the equation for the standard deviation is:

$\sigma_{\mathrm{n}}(\tau)=\sqrt{\sigma_{0}^{2}+\frac{1}{\lambda\left(k_{0}^{\prime \prime} \mathrm{e}^{-\lambda \tau}+1\right)}-\frac{1}{\lambda\left(k_{0}^{\prime \prime}+1\right)}}$

where $\lambda$ must be larger than 0 in this equation. The temporal variations of this parameter under various conditions (linear, non-linear and combined) are illustrated in Fig. 3d.

\subsubsection{Temporal peak standard deviation}

The transformation of the spatial peak width $W_{n}(\tau)$ or $\sigma_{n}(\tau)$ to a temporal $W_{\tau}(\tau)$ or $\sigma_{\tau}(\tau)$ is made simply by dividing the migration speed:

$\sigma_{\tau}(\tau)=\frac{\sigma_{\mathrm{n}}(\tau)}{v_{\mathrm{m}}(\tau)}$

or

$\sigma_{\tau}(\tau)=\sigma_{\mathrm{n}}(\tau)\left(k^{\prime \prime}(\tau)+1\right)$

Examples are illustrated in Fig. 3e. 
Linear isotherm

$k^{\prime \prime}{ }_{0}=2$

$\lambda=0$

(a) decay

constant

$\lambda(\tau)$

(b) variation

of $k^{\prime \prime}(\tau)$
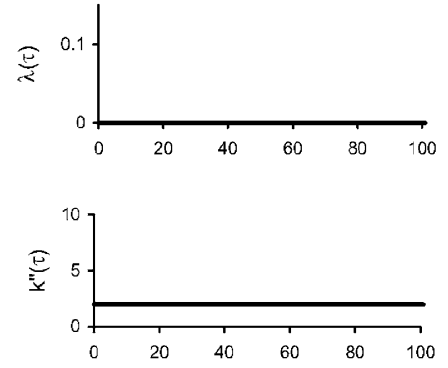

(c) migration

speed

$v_{m}(\tau)$

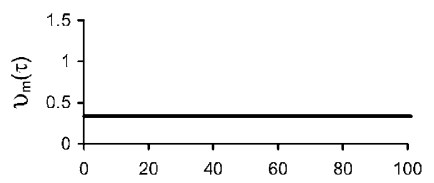

(d) longitudinal

zone

broadening

$\sigma_{n}(\tau)$

(e) temporal

zone

broadening

$\sigma_{z}(\tau)$
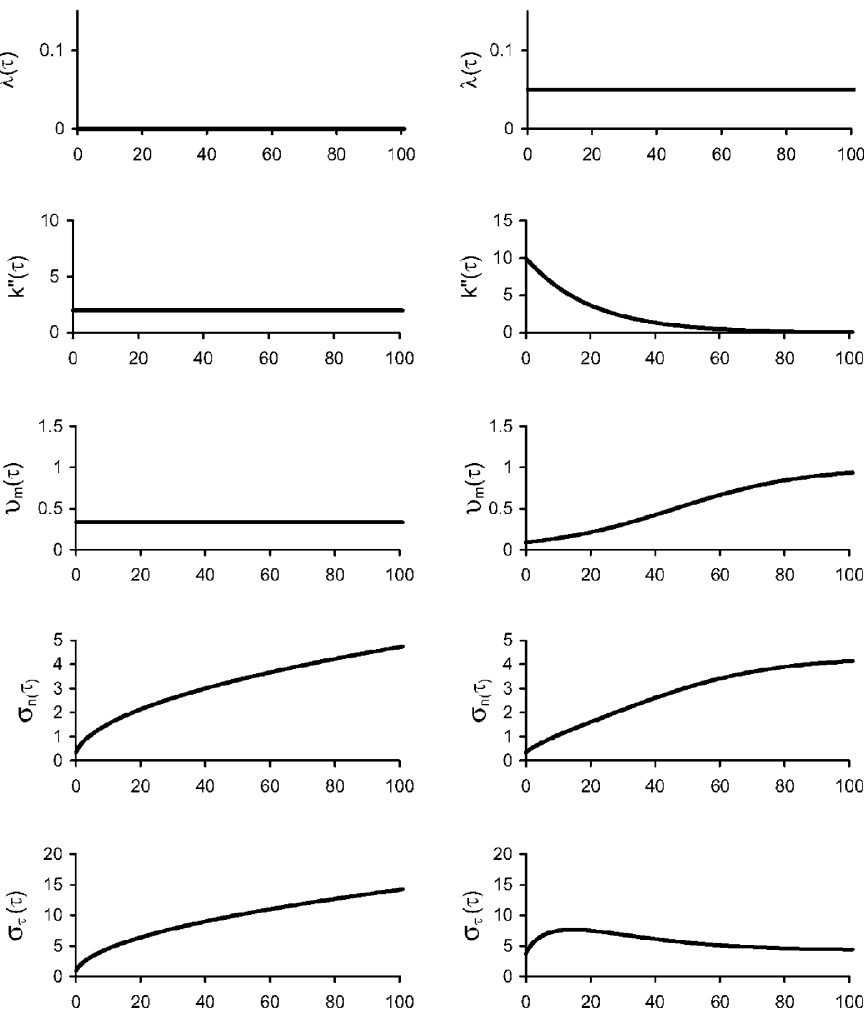

$k^{\prime \prime}{ }_{0}=10$

$\lambda=0.05$
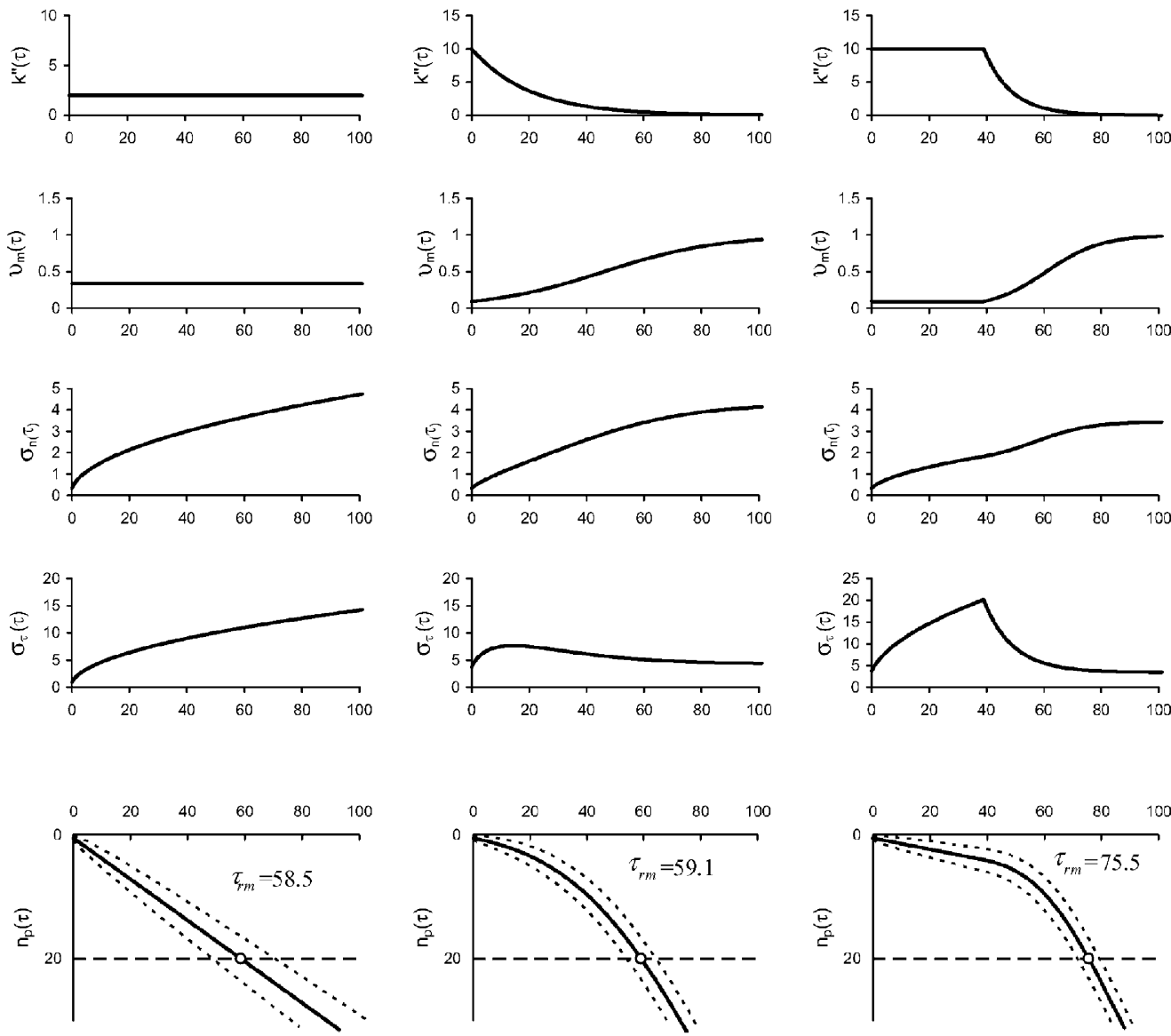

(f) migration

route and

zone

boundaries

$n_{p}(\tau) \pm \sigma_{n}(\tau)$

m

(g) spatial

distribution

when the

peak arrives

at the detector

$\tau=\tau_{r m}$
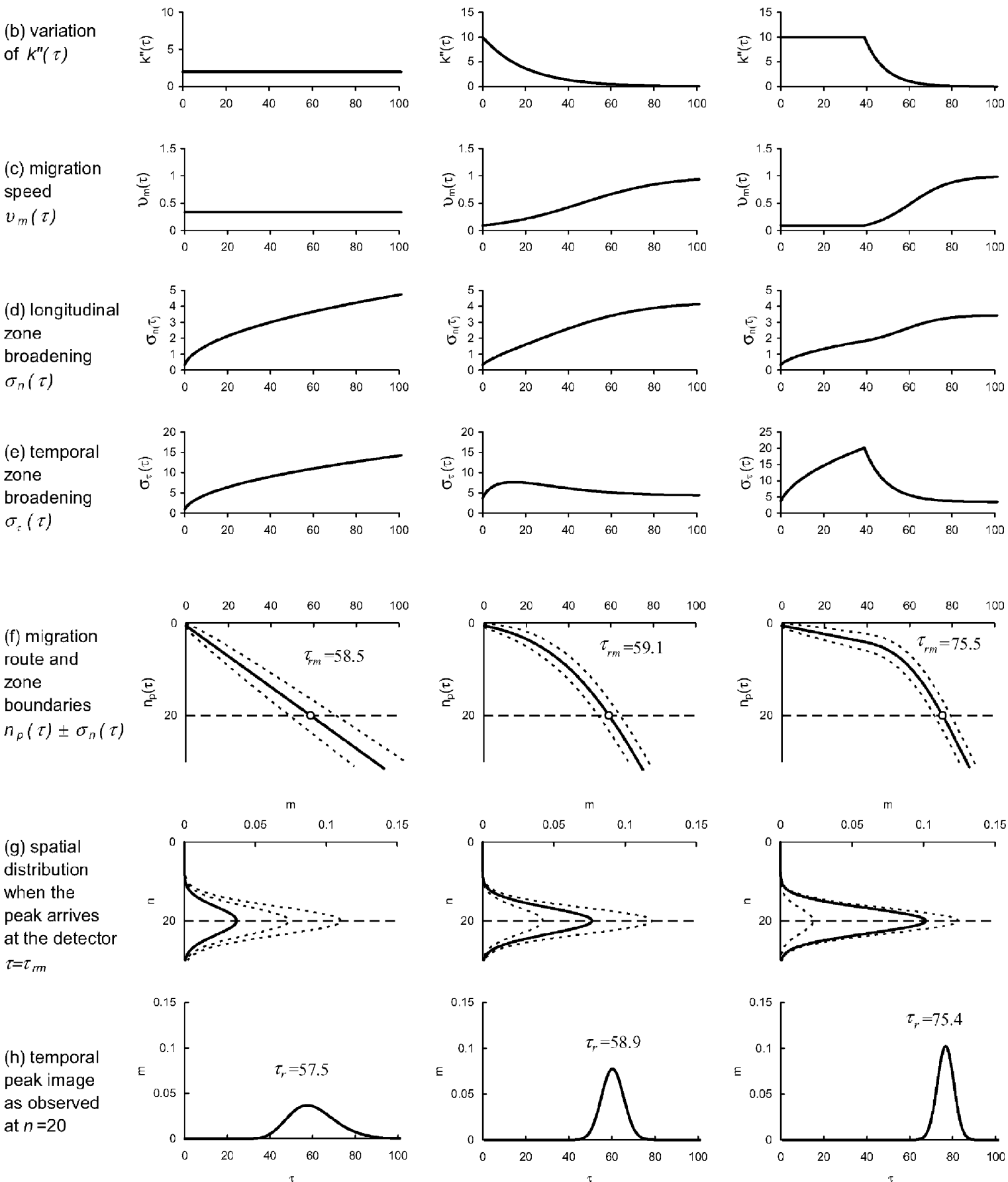

Fig. 3. Graphic display showing examples of the variations of peak parameters during the elution period. The isotherms are linear (left), one-stage non-linear (middle), and stage-wise as a combination of the two (right). (a)-(e) Refer to text, (f) a hypothetic detector is located at $n=20$, (g) the mass patterns are presented for the stationary, mobile phases and the total. Only the mobile phase peak (solid line) can be observed by the detector. (h) the observed temporal peak image, with a peak height close to that of the spatial mobile phase peak, but its position $\tau_{\mathrm{r}}$ is slightly different from $\tau_{\mathrm{rm}}$. 


\subsubsection{Migration map}

The plotting of the moving track of an analyte on the longitudinal-temporal map gives a clear picture of how the sample zone moves and expands during its passage through the column. The curve of $n_{\mathrm{p}}(\tau)$ on the map is the migration route of the mass center; the plotting of $n_{\mathrm{p}}(\tau)+\sigma_{\mathrm{n}}(\tau)$ and $n_{\mathrm{p}}(\tau)-\sigma_{\mathrm{n}}(\tau)$ gives the standard deviation thresholds accompanied with the migration route (see Fig. 3f).

\subsubsection{Mass retention time for a given column length}

For a column of given length $N$, the time required for the mass peak center to arrive at the detector is noted as $\tau_{\mathrm{rm}}$. Thus, under a linear isotherm, it is calculated by (solving Eq. (5)):

$\tau_{\mathrm{rm}}=\frac{N-n_{\mathrm{s}}}{v_{\mathrm{m}}}$

or

$\tau_{\mathrm{rm}}=\left(N-n_{\mathrm{s}}\right)\left(k_{0}^{\prime \prime}+1\right)$

However, under non-linear isotherms, the $\tau_{\mathrm{rm}}$ value is difficult to obtain by an equation, as one may try to solve $\tau_{\mathrm{rm}}$ from Eq. (6) for a given column length $N$ :

$N=n_{\mathrm{s}}+\tau_{\mathrm{rm}}+\frac{1}{\lambda} \ln \left(k_{0}^{\prime \prime} \mathrm{e}^{-\lambda \tau_{\mathrm{rm}}}+1\right)-\frac{1}{\lambda} \ln \left(k_{0}^{\prime \prime}+1\right)$

and there is a direct solution for $\tau_{\mathrm{rm}}$ but a complicated mathematical treatment would be required. Nonetheless, it is not intended to do so because this parameter is not useful in the later convolution process (a hypothetic $\tau_{\mathrm{rm}}^{*}(t)$ should be used for a non-linear isotherm, which will be described in Section 2.2.9). If one must find a value for $\tau_{\mathrm{rm}}$, it would be much easier to estimate it graphically from the migration map (see Fig. 3f).

\subsubsection{Peak area}

The peak area is related to the initial mass $m_{0}$ (or concentration $C_{0}$ ) and size of injection. In the parcel model, the vertical axis is in a "mass" unit and the sample size is defined to be $i=1$, thus the peak areas are $A_{\mathrm{n}}=m_{0} i=m_{0}$ on the longitudinal coordinate and $A_{\tau}=A_{\mathrm{n}} / v$ on the temporal coordinate. Since the flow speed $v$ is also defined to be 1 , for simplicity all peak areas in this paper can be expressed as $m_{0}$.

\subsubsection{Expected arrival time}

This is a hypothetical parameter (not seen in the previous parcel model), quite tricky but the key to the later convolution process. It is used to extract a segment of a curve at time $\tau$ when the mass center has yet to arrive at the detector. This is explained graphically in Fig. 4.

Under a non-linear isotherm, the migration curve is bending downward on the map. If the column length is designated as $N$, at a specific time $\tau$, the distance that has been traveled is $n_{\mathrm{p}}(\tau)$, the distance left to go is $N-n_{\mathrm{p}}(\tau)$. At the

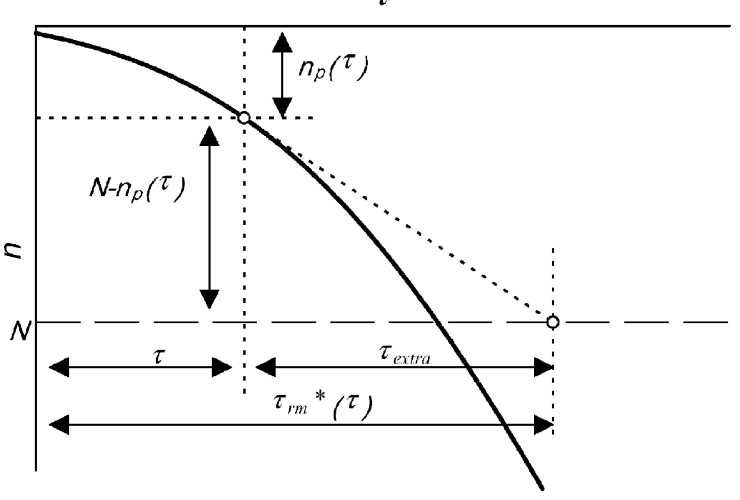

Fig. 4. Calculation of $\tau_{\mathrm{rm}}^{*}(\tau)$ from the migration map. For a given column length $N$, the current peak location at time $\tau$ is $n_{\mathrm{p}}(\tau)$, with a remaining distance of $N-n_{\mathrm{p}}(\tau)$ to reach the end of the column. At the current migration speed $v_{\mathrm{m}}(\tau)=1 /\left(k^{\prime \prime}(\tau)+1\right)$, it needs an extra travel time of $\tau_{\text {extra }}=\left(N-n_{\mathrm{p}}(\tau)\right)\left(k^{\prime \prime}(\tau)+1\right)$ to arrive at the detector. The expected arrival time at a specific time $\tau$ is calculated by $\tau_{\mathrm{rm}}^{*}(\tau)=\tau+\tau_{\text {extra }}$.

current migration speed, $v_{\mathrm{m}}(\tau)=1 /\left(k^{\prime \prime}(\tau)+1\right)$, it would need more time, $\tau_{\text {extra }}(\tau)=\left(N-n_{\mathrm{p}}(\tau)\right)\left(k^{\prime \prime}(\tau)+1\right)$, to arrive at the detector.

Thus, the position of the hypothetical peak summit, or the expected arrival time, denoted as $\tau_{\mathrm{rm}}^{*}(\tau)$, at a given time $\tau$ becomes:

$$
\begin{aligned}
\tau_{\mathrm{rm}}^{*}(\tau)= & \tau+\left[N-\left(n_{\mathrm{s}}+\tau+\frac{1}{\lambda} \ln \left(k_{0}^{\prime \prime} \mathrm{e}^{-\lambda \tau}+1\right)\right.\right. \\
& \left.-\frac{1}{\lambda} \ln \left(k_{0}^{\prime \prime}+1\right)\right]\left(k_{0}^{\prime \prime} \mathrm{e}^{-\lambda \tau}+1\right)
\end{aligned}
$$

For linear cases, the expected arrival time is a constant throughout, and therefore $\tau_{\mathrm{rm}}^{*}(t)=\tau_{\mathrm{rm}}=\left(N-n_{\mathrm{s}}\right)\left(k_{0}^{\prime \prime}+1\right)$ (see Eq. (10)).

\subsection{Mass distribution pattern}

In most cases (as long as the initial $k_{0}^{\prime \prime}$ is not too small or too large, and time is not too short), the mass distribution pattern along a column can be approximated by a limiting Type-I Gaussian equation:

$m_{\text {total }}(n)=\frac{m_{0}}{\sqrt{2 \pi} \sigma_{n}(\tau)} \mathrm{e}^{-\left[\left(n-n_{p}(\tau)\right) /\left(\sqrt{2} \sigma_{n}(\tau)\right)\right]^{2}}$

where $m_{\text {total }}(n)$ is the total mass found along the column length (on $n$ coordinate), $m_{0}$ the initial injected mass value or peak area, $n_{\mathrm{p}}(\tau)$ the position of the peak at a specific time (Eq. (5) or (6)), and $\sigma_{\mathrm{n}}(\tau)$ the standard deviation of the sample zone at the same given time (Eq. (7) or (8)). Since $\tau$ is a given value, the resultant pattern is a symmetrical curve centering at $n_{\mathrm{p}}(\tau)$. The complete expressions of the equation for linear and non-linear isotherms are illustrated in Fig. 5.

The total mass curve can be further divided into stationary and mobile-phase mass curves (i.e. $m_{\mathrm{s}}(n)$ and $m_{\mathrm{m}}(n)$ curves) 
(a) Linear isotherms: Gaussian Type I $\left(n_{s}, m_{o}, k{ }_{o}, \sigma_{o}\right.$ and $\tau$ are given values)

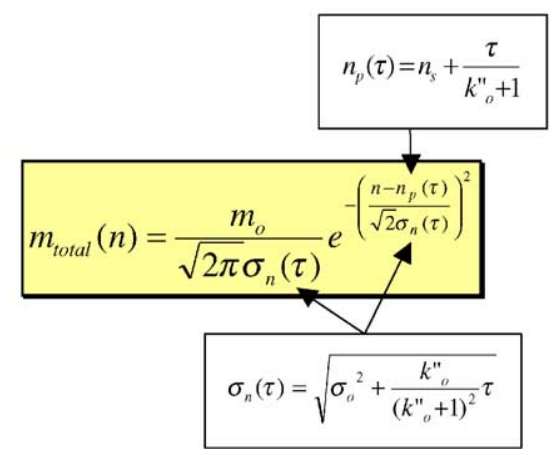

(b) Non-linear isotherms: Gaussian Type $\mathrm{I}\left(n_{s}, m_{o}, k{ }_{o}, \sigma_{o}, \tau\right.$ and $\lambda$ are given values)

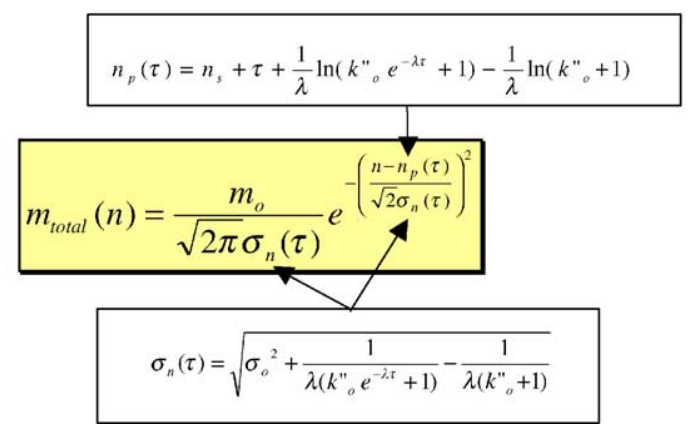

Fig. 5. Construction of Gaussian equations (Eq. (13)) for the mass distribution patterns under (a) linear and (b) non-linear isotherms. Since the peak position and standard deviation are given values, both equations are in the format of limiting Gaussian Type-I. (a) Linear isotherms: Gaussian Type I $\left(n_{\mathrm{s}}, m_{0}, k_{0}^{\prime \prime}, \sigma_{0}\right.$ and $\tau$ are given values). (b) Non-linear isotherms: Gaussian Type $\mathrm{I}\left(n_{\mathrm{s}}, m_{0}, k_{0}^{\prime \prime}, \sigma_{0}, \tau\right.$ and $\lambda$ are given values).

according to the partition equilibrium at time $\tau$. The three curves are synchronized to each other, therefore:

$m_{\mathrm{S}}(n)=\frac{k^{\prime \prime}(\tau)}{k^{\prime \prime}(\tau)+1} m_{\text {total }}(n)$

$m_{\mathrm{m}}(n)=\frac{1}{k^{\prime \prime}(\tau)+1} m_{\mathrm{total}}(n)$

\subsection{Temporal peak}

The temporal view of a spatial pattern (the chromatographic peak) is actually composed of many segmental pieces that are taken from a series of spatial peaks. Thus, at a specific time $\tau$, the detecting signal is a part of a Gaussian equation with a standard deviation $\sigma_{\tau}(\tau)$, and a hypothetical peak position at $\tau_{\mathrm{rm}}^{*}(t)$, at a current migration speed of $v_{\mathrm{m}}(\tau)=1 /\left(k^{\prime \prime}(\tau)+1\right)$. The peak area is the same as the spatial peak if $v=1$.

Thus, the limiting Type-III Gaussian Equation is applied here for the non-linear isotherm, and the general form is given as:

$m_{\mathrm{m}}(\tau)=\frac{m_{0}}{\sqrt{2 \pi} \sigma_{\tau}(\tau)} \mathrm{e}^{-\left[\left(\tau-\tau_{\mathrm{rm}}^{*}(\tau)\right) /\left(\sqrt{2} \sigma_{\tau}(\tau)\right)\right]^{2}}$

were $m_{\mathrm{m}}(\tau)$ is the continuous mass signal recorded for the "mobile phase mass only" along the $\tau$ coordinate, $m_{0}$ the initial injected mass value, $\sigma_{\tau}(\tau)$ the standard deviation at a specific time $\tau$ on the $\tau$ coordinate. The expected arrival time, $\tau_{\mathrm{rm}}^{*}(\tau)$, should be substituted by Eq. (12) for a non-linear isotherm. For linear isotherms, since the $\tau_{\mathrm{rm}}^{*}(\tau)$ is no longer a variable with time (substituted by Eq. (10)), the equation becomes much simpler, and it exactly matches the format of the Type-II Gaussian curve. The complete presentations of Eq. (16) are demonstrated in a systematic format in Fig. 6, for both linear and non-linear isotherms.

\section{Results and discussion}

\subsection{Comparison with parcel model}

The curves generated by Eqs. (13)-(16) are compared with those generated by the discrete parcel matrix [16]. For unifying the column conditions, all parameters are dimensionless, and several values are given as follows: the peak area $m_{0}=1$; the flow speed $v=1$; the longitudinal shift $n_{\mathrm{s}}=0.5$; and the initial standard deviation $\sigma_{0}=0.34$.

In the figures of the following sections, the peaks obtained by the parcel matrix method are marked as discrete circles, while the peaks generated by the proposed equations are plotted in continuous solid lines. 
(a) Linear isotherm: Gaussian Type II ( $N, n_{s}, k^{\prime \prime}$ and $\sigma_{o}$ are given values)

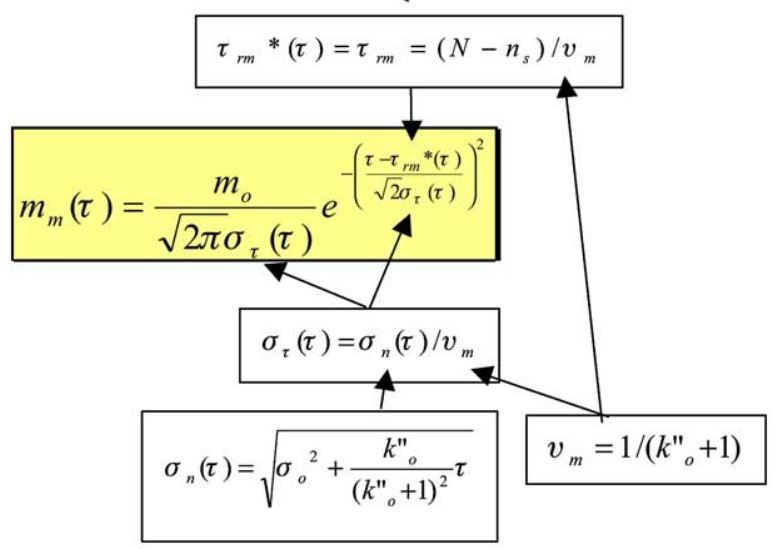

(b) Non-linear isotherm: Gaussian Type III ( $N, n_{s}, k^{\prime \prime}{ }_{o}, \sigma_{o}$ and $\lambda$ are given values)

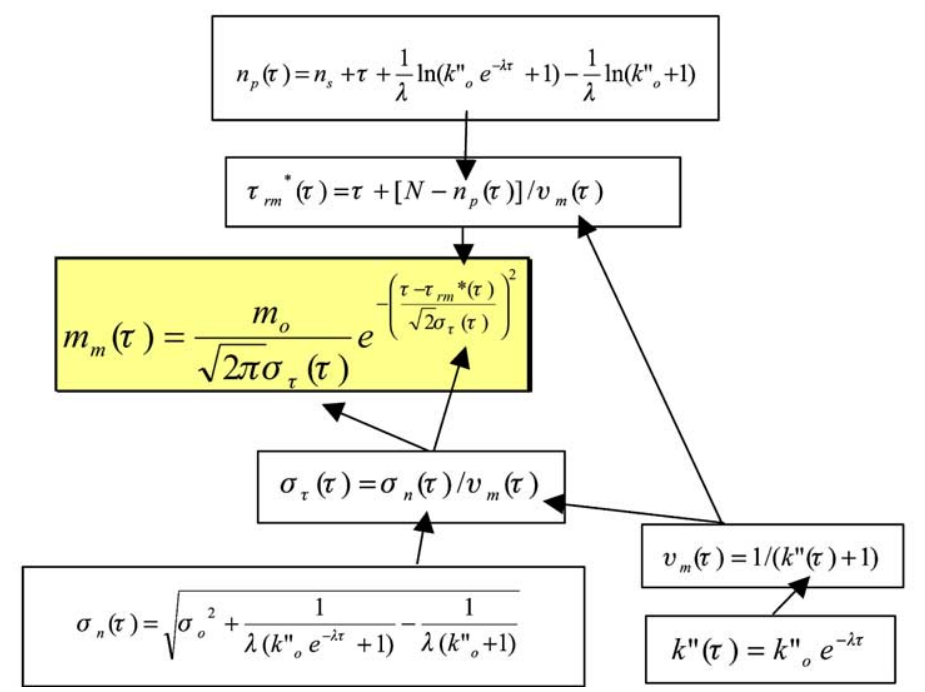

Fig. 6. Temporally-convoluted Gaussian equations (Eq. (16)) for chromatographic peaks under (a) linear and (b) non-linear isotherms. The standard deviation in both equations varies with time. Under a linear isotherm, the peak position is a fixed value, and the equation fits the format of a Gaussian Type-II curve. Under a non-linear isotherm, the peak position is variable with time, and the equation is a typical Gaussian Type-III curve. (a) Linear isotherm: Gaussian Type II $\left(N, n_{\mathrm{s}}, k_{0}^{\prime \prime}\right.$ and $\sigma_{0}$ are given values. (b) Non-linear isotherm: Gaussian Type III ( $N, n_{\mathrm{s}}, k_{0}^{\prime \prime}, \sigma_{0}$ and $\lambda$ are given values).

\subsection{Mass distribution patterns}

The plotting of Eqs. (13)-(15) for the total mass, stationary and mobile-phase masses along a column are demonstrated in Fig. 7. In the linear case (see Fig. 7a), a compound having a partition ratio $k_{0}^{\prime \prime}=5$ is moving following Eq. (5), its moving track shows a straight line on the migration map. The standard deviation is expanding while moving following Eq. (7). The patterns at $\tau=20,40$ and 60 are plotted (in continuous lines) and compared with those obtained by the parcel calculation (in discrete circles). It can be seen that only in the beginning stage, e.g. $\tau=20$, do small deviations occur between the two sets of data (the parcel peaks are slightly not symmetrical). But, as time prolongs, the Gaussian curves match perfectly with the discrete parcel peaks.

Similar situations occur in the non-linear case in Fig. $7 \mathrm{~b}$. When the decay constant is $\lambda=0.02$, a compound with an initial $k_{0}^{\prime \prime}=10$ is migrating following a curvature track of Eq. (6) on the migration map, with an expansion of the standard deviation following Eq. (8). The plottings for Eqs. (13)-(15) give almost identical peak patterns as those obtained by the parcel model.

\subsection{Chromatographic peak}

The plottings of the chromatographic peaks by Eq. (16) are demonstrated in Fig. 8. The migration map is also presented to assist the explanation. A hypothetic detector is located at $n=20$.

Under a linear isotherm, in Fig. 8a, three compounds having $k_{0}^{\prime \prime}$ values of 1, 2 and 5 are given for examples. The mass centers arrive at the detector at $\tau_{\mathrm{rm}}=39,58.5$ and 117 , respectively. Since the migration route is linear, each expected arrival time at any time is a constant $\left(\tau_{\mathrm{rm}}^{*}(\tau)=\tau_{\mathrm{rm}}\right)$. The peak position term in Eq. (16) becomes a fixed value, 


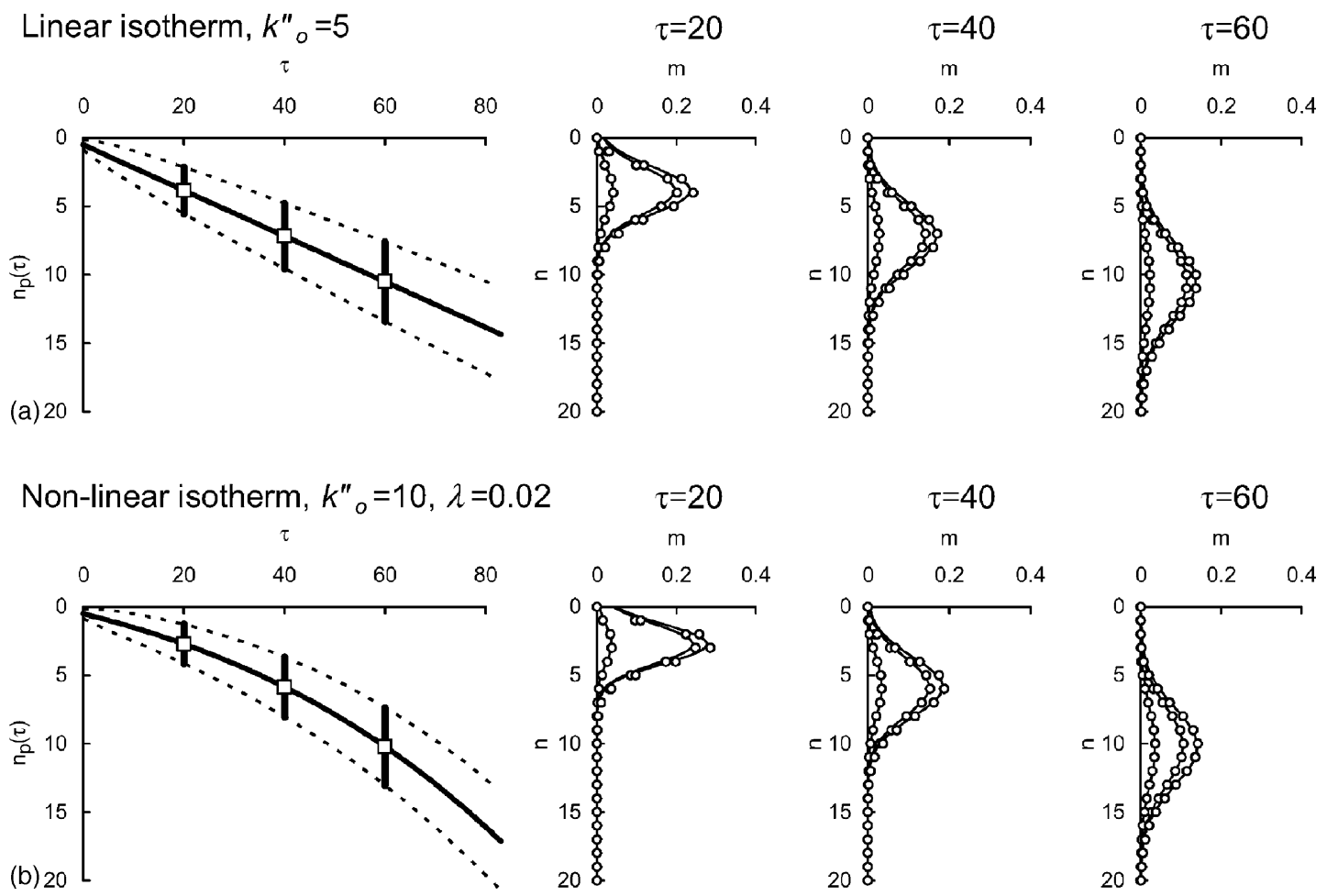

Fig. 7. Migration map demonstrating the peak position and zone broadening (vertical bar) along the $n$ coordinate, together with the mass distribution patterns (total, stationary and mobile phases) at $\tau=20,40$ and 60, respectively. Peaks that are generated by Eqs. (13)-(15) are presented as continuous lines, whereas discrete circles are corresponding data calculated from the parcel model. (a) under linear isotherm, $k_{0}^{\prime \prime}=5$; (b) under a non-linear isotherm, with an initial $k_{0}^{\prime \prime}=10$ and a decay constant $\lambda=0.02$.

leaving only the standard deviation term which is still a function of time. Thus, the temporal image under a linear isotherm fits the condition of the limiting Gaussian Type-II curve. The TCG peaks (plotted in continuous lines) coincide with those obtained by the parcel peaks (plotted in discrete circles).

Under a non-linear isotherm, the situation is different. In Fig. $8 \mathrm{~b}$, three compounds with initial $k_{0}^{\prime \prime}$ values of 1,10 and 50 are migrating under a decay constant of $\lambda=0.02$. The expected arrival time $\left(\tau_{\mathrm{rm}}^{*}(\tau)\right)$ becomes variable with time, and therefore the peak position term in Eq. (16) is not a constant. The minimum $\left(\tau_{\mathrm{rm}}^{*}(\tau)\right)$ values (which reveal the actual arriving time $\tau_{\mathrm{rm}}$ ) were found at $\tau=33.3,90.8$ and 160.1, respectively. The resulting peak starts with a prolonged tailing when $k_{0}^{\prime \prime}$ is low, but gradually turns to a fronting shape. The peak equation becomes the limiting Gaussian Type-III curve. Similar to those of the linear case, the continuous peaks (plotted in continuous lines) match perfectly well with that obtained by the parcel model (discrete circles).

\subsection{Tailing on the temporal coordinate}

Also in Fig. 8, the expansion rate of the standard deviation along the $\tau$ coordinate $\left(\sigma_{\tau}(\tau)\right)$ is particularly pronounced (plotted in the bottom), which can be regarded as an index for the judgment of "peak tailing". Under a linear condition, the sample zone expands proportionally to the square root of time, and all peaks carry a "normal-type" or prolonged tailing. Under a non-linear condition, the sample zone is still expanding spatially, but not necessarily on the temporal coordinate $\left(\sigma_{\tau}(\tau)=\sigma_{\mathrm{n}}(\tau)\left(k^{\prime \prime}(\tau)+1\right)\right)$. If the standard deviation $\sigma_{\tau}(\tau)$ at the detection point is expanding, then the peak carries a prolonged tail. Otherwise, the peak appears to be fronting. The horizontal bars (representing the standard deviation ranges of the peaks at the detector) give a good indication of the symmetry of the tail. For the examples in Fig. 8b, only the first compound gives the prolonged type of tailing, whereas the other two are of the fronting type. This can also be explained that the migration speed is accelerated when passing through the detector, so a "fat" sample zone can be viewed as relatively "slender" in its recorded image.

A tailing index can be defined as:

$\psi\left(\tau_{\mathrm{rm}}\right)=\frac{\mathrm{d} \sigma_{\tau}\left(\tau_{\mathrm{rm}}\right)}{\mathrm{d} \tau}$

where $\psi\left(\tau_{\mathrm{rm}}\right)$ is the index for the peak arriving at the detector. If $\psi>0$, the peak has a prolonged tail; $\psi=0$, it has a Gaussian-like shape; and $\psi<0$, the peak becomes a fronting type. This rule applies only to the one-stage programming of the isotherm. If the isotherm is stage-wise, the peak appearing near the changing point becomes segmental.

\subsection{The temporal shift}

As previously mentioned in the parcel model, the position of a chromatographic peak on the $\tau$ coordinate (denoted 

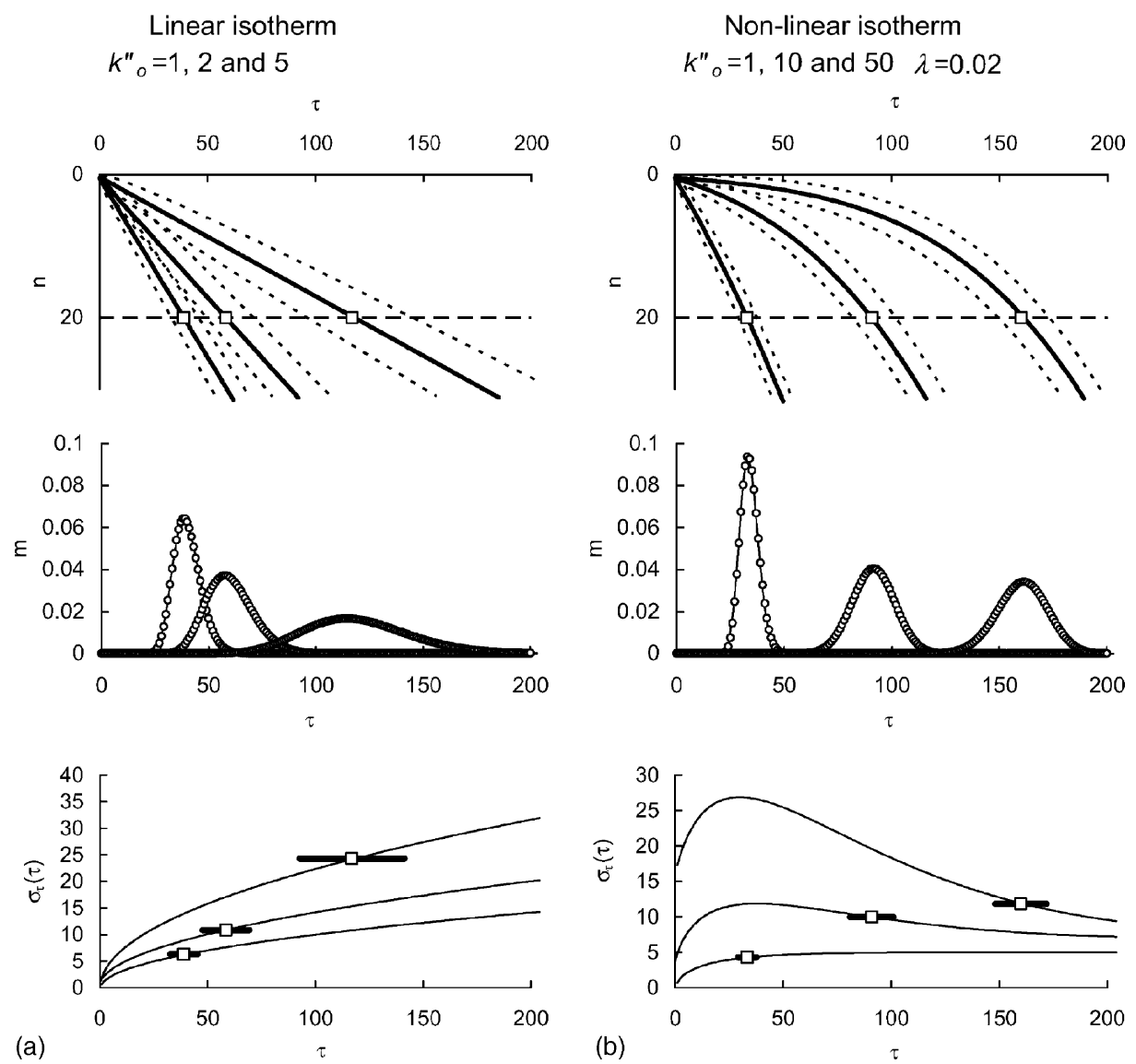

Fig. 8. Demonstration of the temporally convoluted Gaussian (TCG) peaks on the $\tau$ coordinate. (a) $k_{0}^{\prime \prime}=1,2$ and 5 under a linear isotherm; (b) $k_{0}^{\prime \prime}=1$, 10 and 50 under a non-linear isotherm with a decay constant of $\lambda=0.02$. (Top) the migration map with a hypothetical detector at $n=20$, (middle) peaks generated by Eq. (16) are presented by continuous lines, whereas discrete circles are data calculated by the parcel model, (bottom) the temporal standard deviation (horizontal bar) at the peak position serves as an indicator of peak symmetry.

as $\tau_{\mathrm{r}}$ ) may differ from the actual arrival time $\left(\tau_{\mathrm{rm}}\right)$. The peak position shift is obvious and consistent to be $\tau_{\mathrm{rm}}-$ $\tau_{\mathrm{r}}=0.5 k_{0}^{\prime \prime}$ under linear isotherms. For the example shown in Fig. 3, when $k_{0}^{\prime \prime}=2$, the arrival time is $\tau_{\mathrm{rm}}=58.5$, and the peak summit appears at $\tau_{\mathrm{r}}=57.5$ on the recorder. For non-linear cases, the shift can also be approximated by $0.5 k^{\prime \prime}\left(\tau_{\mathrm{rm}}\right)$, but this relationship does not completely apply under a step-wise programmed isotherm. The shift might turn to the other direction, i.e. the peak summit may appear near or even after $\tau_{\mathrm{rm}}$, depending on the given conditions.

\subsection{Recursion-assisted TCG curve}

The TCG peak equation shown in Fig. 6 is quite complicated. It can be even more complicated if the isotherm is separated into several stages during the operation. For the convenience of running such a multi-stage condition on a computer worksheet, a recursion technique is recommended. In other words, the mathematical terms in the TCG equation can be regarded as "subroutines", which are calculated separately in a recursion manner. The only condition is that the time step number must not be too small.
The procedures for setting up the recursion-assisted TCG on Microsoft Excel are demonstrated in Fig. 9. The recursion formulae are:

$k^{\prime \prime}(\tau)=k^{\prime \prime}(\tau-1) \times(1-\lambda(\tau))$

$n_{\mathrm{p}}(\tau)=n_{\mathrm{p}}(\tau-1)+\frac{1}{\left(k^{\prime \prime}(\tau)+1\right)}$

$\sigma_{n}(\tau)=\sqrt{\sigma_{\mathrm{n}}(\tau-1)^{2}+\frac{k^{\prime \prime}(\tau)}{\left(k^{\prime \prime}(\tau)+1\right)^{2}}}$

$\sigma_{\tau}(\tau)=\sigma_{\mathrm{n}}(\tau) \times\left(k^{\prime \prime}(\tau)+1\right)$

$\tau_{\mathrm{rm}}^{*}(\tau)=\tau+\left(N-n_{\mathrm{p}}(\tau)\right) \times\left(k^{\prime \prime}(\tau)+1\right)$

These formulae are also presented in the Excel format in Fig. 9. They provide the basic parts to build a Gaussian curve along time coordinate. In this way, if the time number is not too small, one can easily produce a peak shape which is compatible to that produced by a discrete parcel matrix or a completely continuous equation.

The recursion-assisted TCG curve (RATCG) takes advantages of both the TCG and the parcel model in producing the temporal peak under multi-stage programming: 


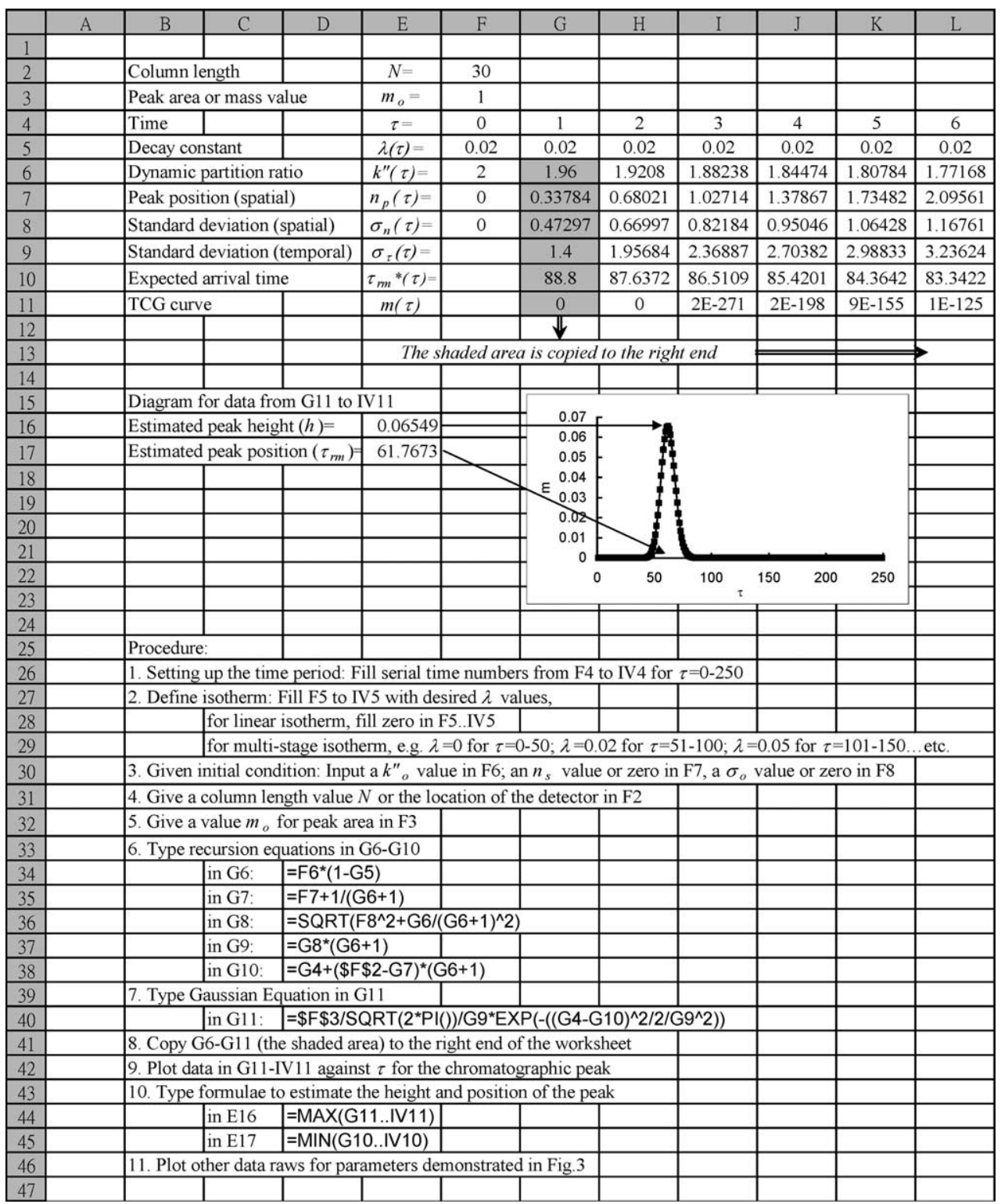

Fig. 9. Illustration of the recursion-assisted technique for plotting the TCG curve on the Excel worksheet. It is probably the easiest way to draw a single chromatographic peak under a multi-stage isotherm.

(1) It does not need to type a long TCG equation for the calculation.

(2) It fits all types of isotherm programming, including linear, one-stage non-linear and multi-stage isotherms. The decay constant can be set zero for all cases (i.e. $\lambda=0$ is allowed in the recursion calculation).

(3) It does not need to produce a vast parcel matrix (two-dimensional) for a longer column.

Since the RATCG curve is probably the simplest way to produce a chromatographic peak, one may generate many peaks on the same worksheet and combine them to create a chromatogram. For further examples with Excel one may refer to a recent textbook by de Levie [18].

\subsection{Multi-stage isotherm}

Nearly all GC applications involve the setting up of a temperature program for the optimal separation of a group of components. Thus, the program may consist of a starting linear isotherm stage for those very mobile components, followed by the increase of temperature to separate sequentially compounds with various $k_{0}^{\prime \prime}$ values. The elevation can be made in different stages, depending on the group characteristics. At the end, a terminal stage might be given to accelerate those very retarding compounds to leave the column. In real applications, the temperature programming varies from case to case, depending on the nature of the sample, as well as the conditions of instrumentation. 
Case 1
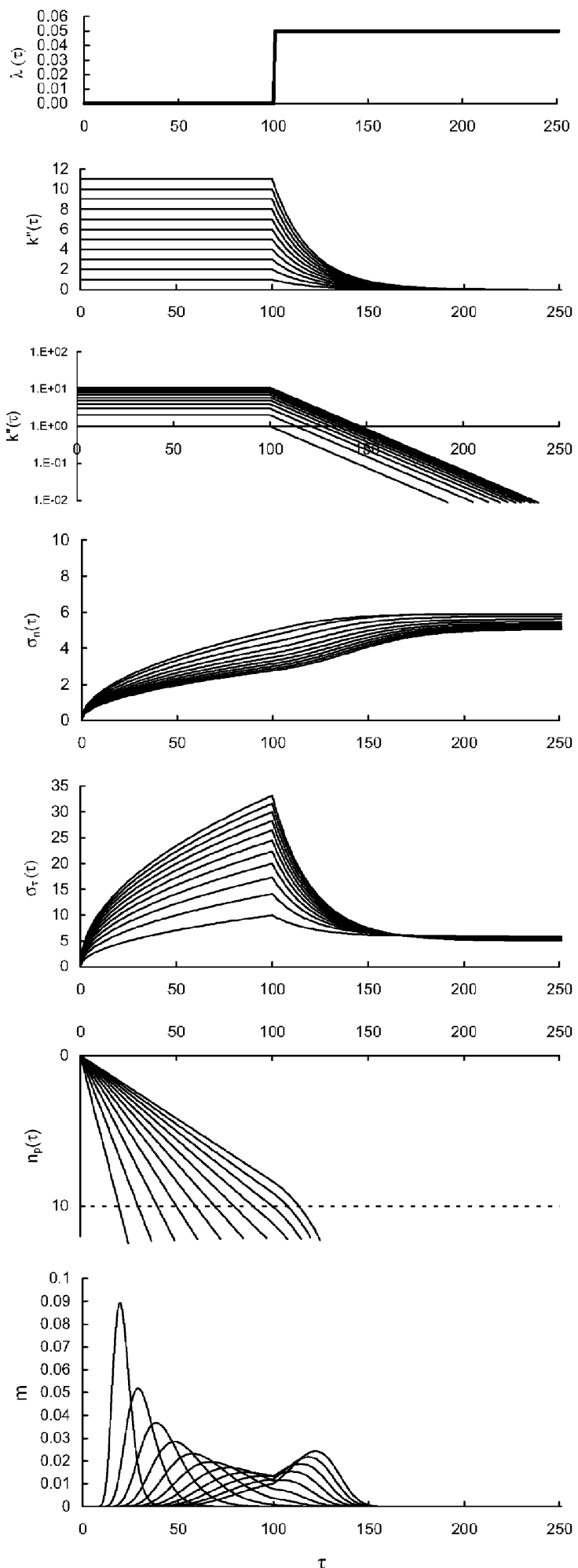

Case 2
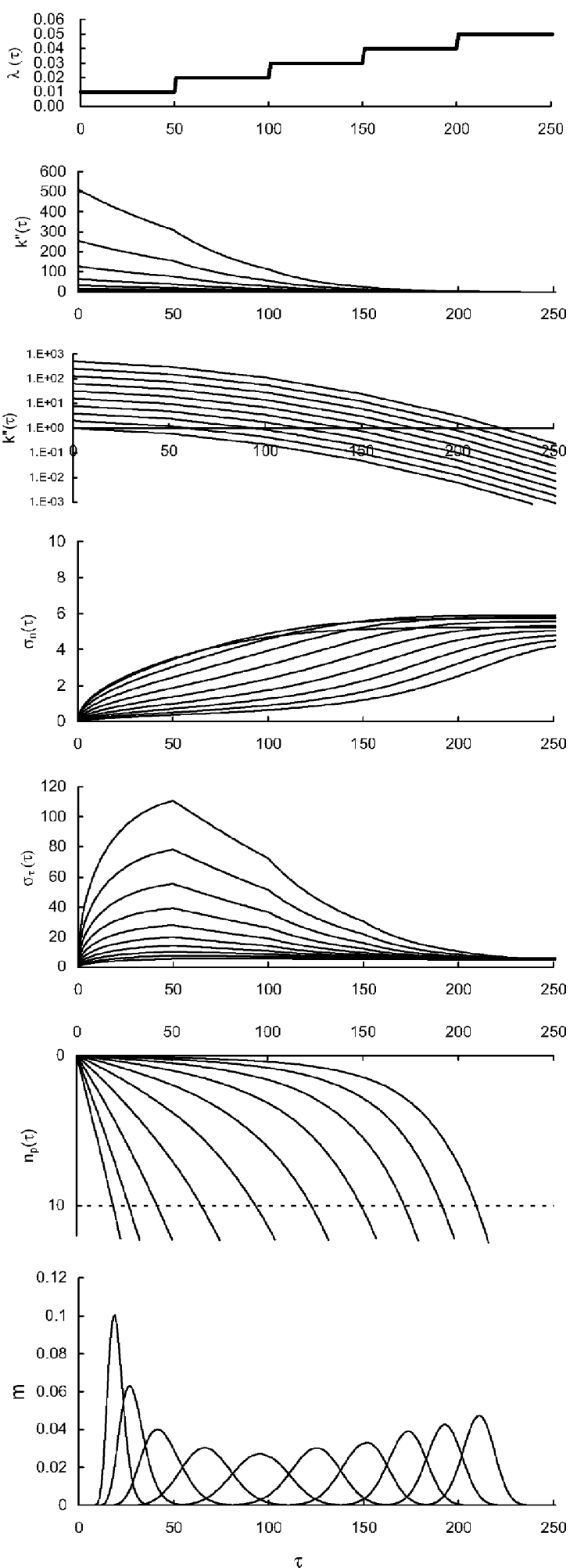

Fig. 10. Overlapped plot of a group of peaks under multi-stage isotherms drawn by the recursion-assisted temporally convoluted Gaussian curves (RATCG) on the Excel worksheet. The detector is located at $n=10$. Case-1, two-stage programming, ten components with initial $k_{0}^{\prime \prime}$ values of $1,2,3,4,5$, 6, 7, 8, 9 and 10, respectively. Case-2, five-stage programming, initial $k_{0}^{\prime \prime}$ values are 1, 2, 4, 8, 16, 32, 64, 128, 256 and 512, respectively. The initial $k_{0}^{\prime \prime}$ values for both Cases 3 and 4 are 1, 2, 4, 8, 16, 32, 64, 128, 256 and 512, respectively. Case 3, multi-stage programming with two non-linear intervals. The peak shape follows a t-f-t-f-t sequence, with obvious segmental shapes when near the changing points. Case 4, multi-stage programming with two linear intervals and a terminal stage. The peak shape follows a t-f-t-f-G sequence. In the ending period, peaks are nearly Gaussian. 
Case 3
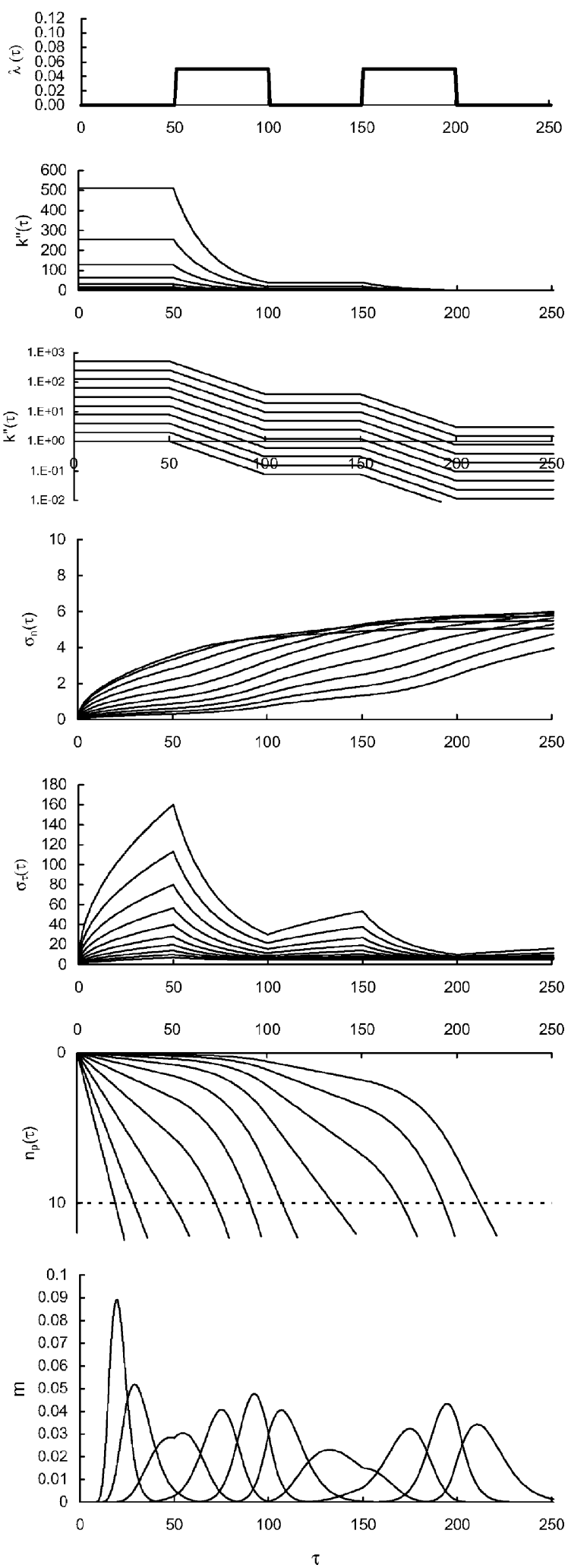

Case 4
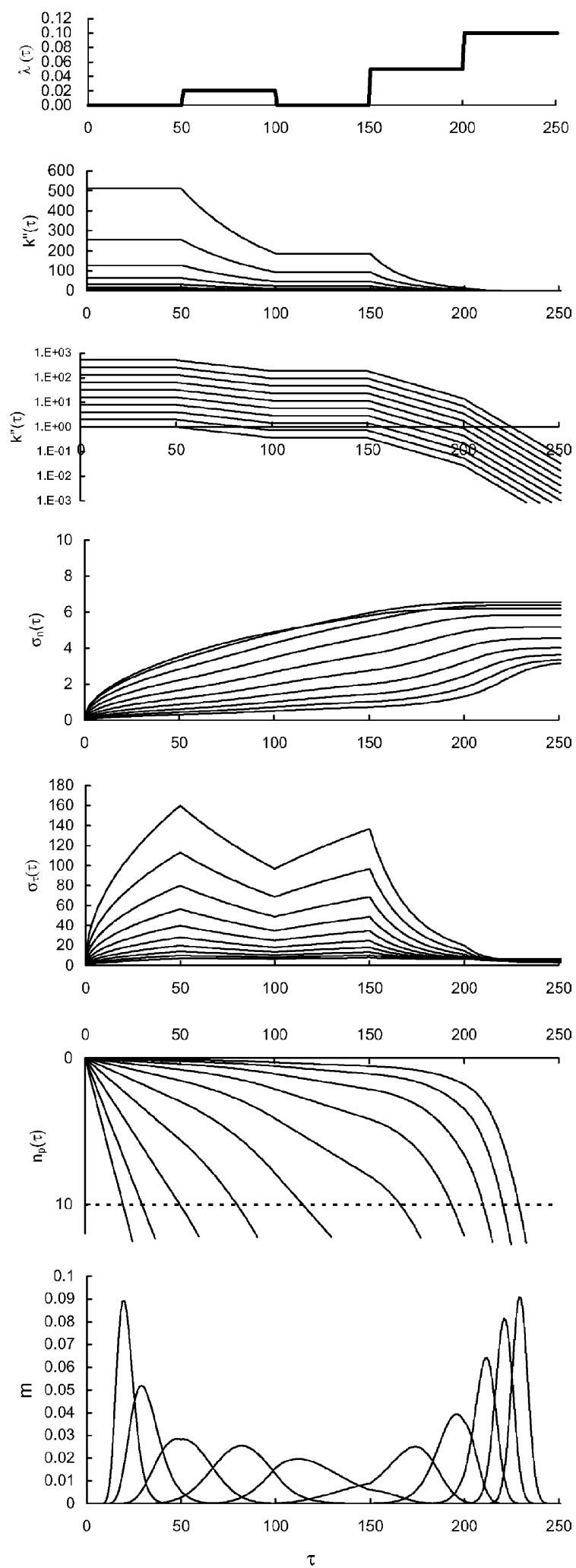

Fig. 10. (Continued). 
Using the RATCG equation as a tool, one may readily simulate different situations on a computer worksheet. Several examples are illustrated in Fig. 10, in which the time span is $\tau=0-250$ and a hypothetic detector is located at $n=10$.

Case 1 (Two stage programming). Ten compounds with initial $k_{0}^{\prime \prime}$ values of $1,2,3,4,5,6,7,8,9$ and 10 are tested. The isotherm is constant $(\lambda=0)$ from $\tau=0-100$, and starts to decrease with a decay constant of $\lambda=0.02$ after $\tau=$ 101. In addition to the resultant chromatographic curves, the migration map and other peak parameters are also plotted. In the linear range $\tau<100$, the peaks are typical tailing shapes. After the changing point, the peaks become segmental, and the appearance changes from tailing to segmental fronting.

Case 2 (Five-stage programming). The initial $k_{0}^{\prime \prime}$ values for the ten tested compounds are 1, 2, 4, 8, 16, 32, 64, 128, 256 and 512, respectively. The programming starts with a $\lambda=$ 0.01 , which changes to $0.02,0.03,0.04$ and 0.05 at $\tau=51$, 101,151 and 201, respectively. Only the three leading peaks carry a prolonged tail, whereas all others turn to be slightly fronting. The gradual decrease of the $k^{\prime \prime}(\tau)$ value leads to a much smoother looking peak shape.

Case 3 (Five-stage programming with non-linear intervals). The $k_{0}^{\prime \prime}$ values are the same as in Case 2. The program is basically linear, but with two accelerating periods between $\tau=51-100$ and $\tau=151-200$. Spatially, the sample zones are all expanding with time; but temporally, the peak widths shrink during the non-linear isotherm range, which can be identified by the $\sigma_{\tau}(\tau)$ plot. The peak type follows the sequence: tailing-fronting-tailing-fronting-tailing (t-f-t-f-t in brief). At the changing points, some peaks appear to be segmental.

Case 4 (Five-stage programming with intervals and a terminal stage). Similar to Case 3, but a terminal stage $(\lambda=0.1$ after $\tau=201$ ) is applied to release those having high $k_{0}^{\prime \prime}$ compounds from the column. This action leads to a narrower peak width and a higher peak height in the ending period. The last peak looks very symmetrical, judging also from the $\sigma_{\tau}(\tau)$ plot. The peak type follows a t-f-t-f-G trend ( $\mathrm{G}$ for Gaussian).

All of the RATCG diagrams are almost identical to those created by the parcel matrix [16] or by the continuous equations (calculated for five separate stages).

\subsection{Diffusion modification}

The TCG equation can be further modified to include the longitudinal dispersion-diffusion effect, which is also a zone broadening factor. Diffusion will increase the standard deviation, but will not affect the position of a peak during the migration. Thus, the spatial variance can be written as:

$\sigma_{\mathrm{n}}(\tau)^{2}=\sigma_{0}^{2}+\sigma_{\text {retention }}(\tau)^{2}+\sigma_{\text {diffusion }}(\tau)^{2}$

and the diffusion term is defined as:

$\sigma_{\text {diffusion }}(\tau)=\sqrt{2 D \tau}$

where $D$ is a dimensionless diffusion coefficient in this study. It is a constant under a linear isotherm, but can be variable with time under a non-linear isotherm. In the latter case it should be denoted as $D(\tau)$.

Accordingly, under a linear isotherm, the spatial peak standard deviation as stated in Eq. (7) becomes:

$\sigma_{\mathrm{n}}(\tau)=\sqrt{\sigma_{0}^{2}+\frac{k_{0}^{\prime \prime}}{\left(k_{0}^{\prime \prime}+1\right)^{2}} \tau+2 D \tau}$

whereas under a non-linear isotherm, Eq. (8) can be re-written as:

$\sigma_{\mathrm{n}}(\tau)=\sqrt{\sigma_{0}^{2}+\frac{1}{\lambda\left(k_{0}^{\prime \prime} \mathrm{e}^{-\lambda \tau}+1\right)}-\frac{1}{\lambda\left(k_{0}^{\prime \prime}+1\right)}+2 D(\tau) \tau}$

When either of these two modified terms is substituted into the spatial peak equation (Eq. (13)), the peak pattern is still Gaussian; only the peak height is relatively lower, and the width slightly wider or fatter. This also applies to the temporally convoluted peaks (Eq. (16)). The temporal appearance will be slightly altered, but the trend of skewness, the peak position and the migration route remain the same. As a result, it can be concluded that the longitudinal dispersion-diffusion is not a major factor that causes peak asymmetry (it diminishes the asymmetry).

\section{Conclusion}

Attempts of using the Gaussian function to simulate chromatographic peak shapes have been extensively described, and many modified Gaussian equations were proposed to fit the observed peak shapes [8-14]. However, complete success cannot be reached without endorsing the temporal distortion effect, which is still a rather intangible or abstract concept to most chromatographers. The implementation of the hypothetic parameter $\tau_{\mathrm{rm}}^{*}(\tau)$ is an important breakthrough, which offers a quick mathematical process for the axial transformation, especially under a non-linear isotherm, with sufficient physical meanings. After proper convolution treatments, the Gaussian function is still the backbone of all types of peaks, and the proposed TCG equation can be used as a solid foundation for further explanation of peak shape problems. However, before it can be applied to simulate a real chromatogram, additional modifications to include the diffusion and extra column effects would be necessary.

It is interesting to note that, the entire convolution concept is still based on the simple equilibrium of plate theory, i.e. 
$k^{\prime \prime}=m_{\mathrm{s}} / m_{\mathrm{m}}$, without considering the complex Langmuir isotherms or the multi-layer diffusion mechanisms. Yet, it produces all types of peaks, which would have been normally regarded in the past as the extra column effects or parabolic flow profile. This leads scientists to re-consider that these spatial effects, although proven to exist, may not be the exclusive reasons for peak asymmetry. Further experimental clarification between the spatial and temporal effects is especially encouraged.

\section{Acknowledgements}

The author would like to thank K. Ronning and Y.I. Chang for their kind criticism of the manuscript. Suggestions by two anonymous reviewers are also appreciated. This work is partially sponsored by the National Science Council (Taipei) under the grant no. NSC91-2611-M-002-009 and NSC92-2611-M-002-022.

\section{References}

[1] S. Golshan-Shirazi, G. Guiochon, in: F. Dondi, G. Guiochon (Eds.), Theoretical Advancement in Chromatography and Related Separation Techniques, Kluwer Academic, Dordrecht, 1991.
[2] D.A. Skoog, F.J. Holler, T.A. Nieman, 5th ed., Principles of Instrumental Analysis, Saunders College, Philadelphia, 1998.

[3] K. Robards, P.R. Haddad, P.E. Jackson, Principles and Practice of Modern Chromatographic Methods, Academic Press, London, 1994.

[4] J.T. Vanderslice, K.K. Stewart, A.G. Rosenfeld, D.J. Higgs, Talanta 28 (1981) 11

[5] A. Prüß, C. Kempter, J. Gysler, T. Jira, J. Chromatogr. A 1016 (2003) 129.

[6] C.E. Evans, V.L. McGuffin, Anal. Chem. 63 (1991) 1393.

[7] B. Lin, G. Guiochon, Theory and Modeling of Nonlinear Chromatography, Elsevier, Amsterdam, 2003.

[8] V.B. Di Marco, G.G. Bombi, J. Chromatogr. A 931 (2001) 1.

[9] J.P. Foley, J.G. Dorsey, J. Chromatogr. Sci. 22 (1984) 40.

[10] J.R. Torres-Lapasió, J.J. Baeza-Baeza, M.C. García-Alvarez-Coque, Anal. Chem. 69 (1997) 3822.

[11] J. Li, Anal. Chem. 69 (1997) 4452.

[12] P. Nikitas, A. Pappa-Louisi, A. Papageorgiou, J. Chromatogr. A 912 (2001) 13.

[13] J. Li, J. Chromatogr. A 952 (2002) 63.

[14] R.D. Caballero, M.C. García-Alvarez-Coque, J.J. Baeza-Baeza, J. Chromatogr. A 954 (2002) 59.

[15] S.C. Pai, J. Chromatogr. A 950 (2002) 271.

[16] S.C. Pai, J. Chromatogr. A 988 (2003) 233.

[17] S.C. Pai, C.C. Chern, L.Y. Chiao, J. Chromatogr. A 1016 (2003) 125.

[18] R. de Levie, How to Use Excel in Analytical Chemistry and in General Scientific Data Analysis, Cambridge University Press, 2001. 